\title{
Electrically Small Superconducting Antennas With Bandpass Filters
}

\author{
S. Oda, S. Sakaguchi, H. Kanaya, R. K. Pokharel, and K. Yoshida
}

\begin{abstract}
In order to reduce the size of a wireless system, we have proposed the design formulas for an electrically small antenna (ESA), i.e. an antenna whose dimension is much smaller than a wavelength, with a miniaturized matching circuit which connects to a $50 \Omega$ external circuit. Theoretical performances of the slot dipole antenna with the impedance matching circuit designed by the present theory are studied by the electrical circuits using transmission lines (Transmission Line Model) as well as the electromagnetic (EM) field simulator. We designed a slot dipole antenna with the aid of the simulations using the electrical circuits as well as the EM field simulator. The size of the designed antenna including the matching circuit is $4.1 \mathrm{~mm} \times 1.9 \mathrm{~mm}$ on $\mathrm{MgO}$ substrate with relative permittivity of 9.6 at the center frequency of $5 \mathrm{GHz}$, and the designed fractional bandwidth is $13 \%$ @ $R L$ (Return Loss) = $-3 \mathrm{~dB}$. In order to demonstrate the theory, we also carried out experiments on the slot dipole antenna with the matching circuits of pole number $n=2$ using high temperature superconductors YBCO on MgO substrates in the $5 \mathrm{GHz}$ band.
\end{abstract}

Index Terms-Bandpass filter, Coplanar waveguide, miniaturized impedance matching circuit, slot loop antenna.

\section{INTRODUCTION}

D URING recent years, miniaturized planar antennas are essential to radio communication devices such as wireless LAN, MIMO and RF-ID in information society, because the size of antenna often has a great influence on the whole size of a wireless system. There are many reports on electrically small antennas (ESA), i.e., antennas whose dimension is much smaller than the wavelength of interest [1]-[5].

The characteristic of antenna is closely concerned with its dimension. A small antenna is sensitive to the conductor resistance because of its low radiation resistance, and the decrease of the radiation efficiency often makes serious problem. High temperature superconductor (HTS) antennas, whose electrode resistance is negligibly small, are useful for miniaturization [6]-[11]. In order to realize an ESA, it is also necessary to realize a broadband matching circuit that compensates the narrow bandwidth

Manuscript received August 29, 2006. This work was supported in part by a Grant-in-Aid for Scientific Research (B) from the Japan Society for the Promotion of Science (JSPS) and by a grant of Fukuoka project in the Cooperative Link of Unique Science and Technology for Economy Revitalization (CLUSTER) of Ministry of Education, Culture, Sports, Science and Technology (MEXT).

The authors are with the Department of Electronics, Graduate School of Information Science and Electrical Engineering, Kyushu University, Motooka747, Nishi-ku, Fukuoka 819-0395, Japan (e-mail: yoshida@ed.kyushu-u.ac.jp).

Color versions of one or more of the figures in this paper are available online at http://ieeexplore.ieee.org.

Digital Object Identifier 10.1109/TASC.2007.898273

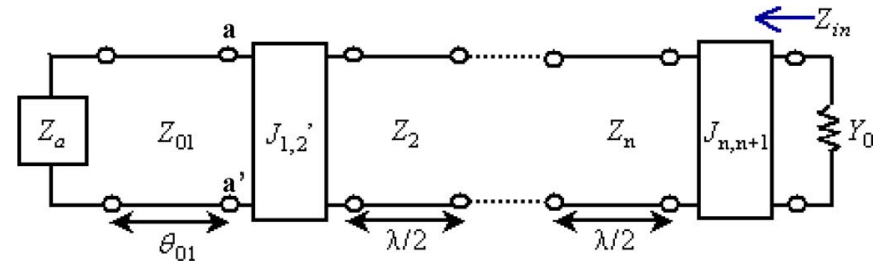

Fig. 1. Equivalent circuit of the antenna with n-pole bandpass filter.

peculiar to small antennas with an low impedance. We must attain large matching ratios to connect to semiconductor devices with high impedances.

Coplanar waveguide (CPW) fed antennas have advantages such as low attenuation, less distortion, and uni-planar configuration. Furthermore, this feature provides much convenience in system integration for that the input of the antennas can directly connect to the CPW outputs of semiconductor devices. Many slot antennas suitable for a CPW-fed have been reported [3]-[5].

In our previous works, we designed the slot dipole antenna whose length is one wavelength with a bandpass filter using CPW lines [8], [9] which acts as an impedance matching circuit as well. In order to reduce the total size of antenna, the slot loop antenna was also designed whose length is one wavelength in circumference [10], and it was integrated to a low noise amplifier with the matching circuit for interconnecting them [11].

In this paper, we designed a slot dipole antenna whose dimension is much smaller than one wavelength with aid of EM-simulator and carried out the experiments on the slot dipole antenna with 2-poles bandpass filter, whose total size is $4.1 \mathrm{~mm}$ $\times 1.9 \mathrm{~mm}$, using high temperature superconductors YBCO thin film on the substrate with relative permittivity of 9.6 , in the 5.0 $\mathrm{GHz}$ band.

\section{THE THEORY OF ESA With BANDPASS FiLTERS}

Fig. 1 shows the circuit model of the proposed ESA with $n$-pole bandpass filter, which was obtained by modifying the design theory given in [10], [11]. In the figure, $Z_{a}=R_{a}+\mathrm{j} X_{a}$ and $Z_{0}(=50 \Omega)$ represent the internal impedance of the antenna and that of the external port. In the present design, we use slot dipole antennas whose length is much smaller than a wavelength, whose reactance $X_{a}$ is assumed to behave inductively. 
The proposed theoretical values for the electrical length and characteristic impedance of transmission line $\left(\theta_{01}, Z_{01}\right)$ and $J$-parameters of $J$ inverters $\left(J_{i}, i+1\right)$ [12] are given by [13]

$$
\begin{aligned}
\theta_{01} & =\frac{1}{2} \cdot \operatorname{Sinc}^{-1}\left(\frac{Q_{a}}{2 Q_{e 1}-Q_{a}}\right) \\
Z_{01} & =\left.X_{a}\right|_{\omega=\omega_{0}} \cdot \tan \theta_{01} \\
J_{1,2}^{\prime} & =w \sqrt{\frac{b_{1}^{\prime} b_{2}}{g_{1} g_{2}}} \\
J_{i, i+1} & =w \sqrt{\frac{b_{i} b_{i+1}}{g_{i} g_{i+1}}} \quad(i=2,3, \ldots, n),
\end{aligned}
$$
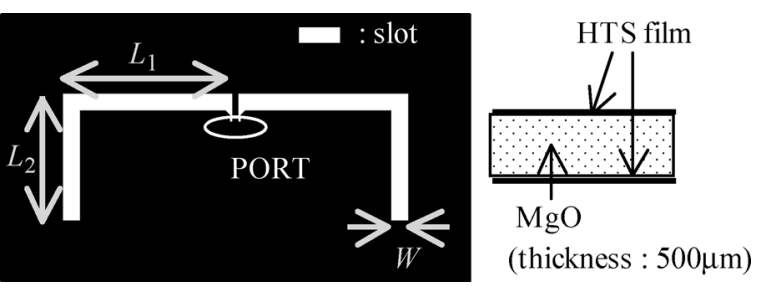

Fig. 2. Structure of the slot dipole antenna.

(a)

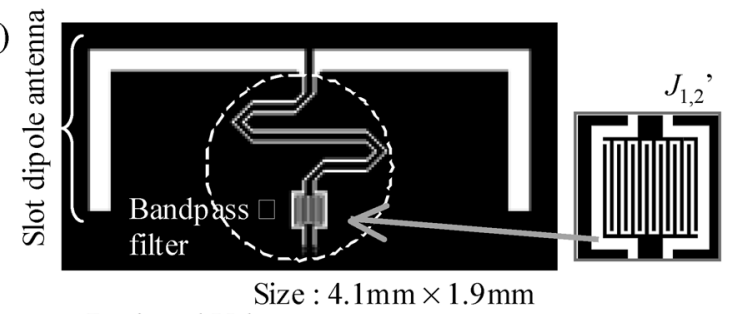

Designed Value

$$
\begin{aligned}
Q_{e 1} & =\frac{g_{0} g_{1}}{w} \\
Q_{a} & =\frac{\left.X_{a}\right|_{\omega=\omega_{0}}}{R_{a}} \\
b_{1}^{\prime} & =\frac{\pi}{4 Z_{01}} \\
b_{i} & =\frac{\pi}{2 Z_{i}} \quad(i=2,3, \ldots, n) \\
\operatorname{Sinc}(x) & =\frac{\operatorname{Sin} x}{x}
\end{aligned}
$$

where $g_{i}(i=1,2, \ldots, n)$ is the normalized filter element, $w$ is the normalized bandwidth [8], $Q_{a}$ and $Q_{e 1}$ are the unloaded $\mathrm{Q}$ factor of the antenna and the external Q factor, and $b_{i}(i=$ $2,3, \ldots, n)$ is the susceptance slope parameter. The equations for $\theta_{01}, Z_{01}$ and $b_{1}$ 'were obtained with the condition $\left|Z_{a}\right| \ll$ $Z_{0}$, i.e., the antenna impedance is sufficiency low.

It is noted that, because the present design formulas are derived from the conventional design theory of Chebyshev BPF [12], and it is possible not only to match the impedance but also to adjust the bandwidth.

\section{The Design of Slot Dipole Antennas}

Antennas with a bandpass filter were designed using HTS films on $\mathrm{MgO}$ substrate in $5 \mathrm{GHz}$ band. In the present design, we use a slot dipole antenna whose length is much smaller than the wavelength, as shown in Fig. 2. In order to reduce the total size of antenna, the end of each slot, which is assumed to slightly contribute to radiation, is bended. $R_{a}=3.78 \Omega$ and $X_{a}=$ $32.08 \Omega$ are obtained for the length of slot $L_{1}=2000 \mu \mathrm{m}$, $L_{2}=1500 \mu \mathrm{m}$ and the width of slot $W=200 \mu \mathrm{m}$, by EM field simulator.

Figs. 3(a) and 3(b) show the layouts and the designed values of the slot dipole antennas with a bandpass filter, in the case of $n=1$ and $n=2$, respectively. In these design, $J$-inverters consist of interdigital gaps in the center signal lines of CPW. In order to keep the total size small even if the number of pole increases, CPW lines are of meandering geometry.

Figs. 4 and 5 show the frequency dependences of the reflection $S_{11}$ which are calculated by EM-field simulator and transmission line model in the case of $n=1$ and $n=2$, respectively.

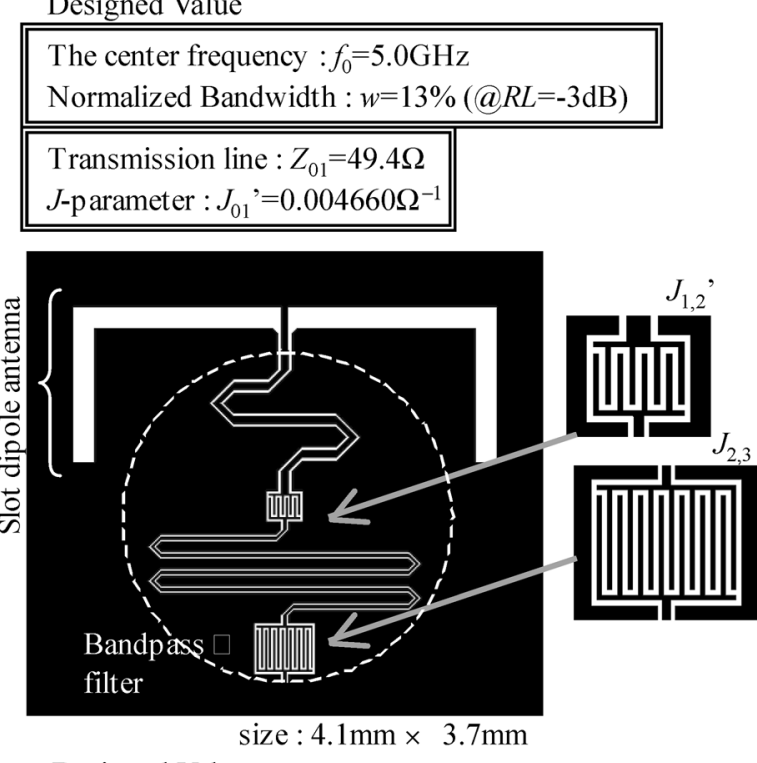

Designed Value

\begin{tabular}{||l||}
\hline \hline The center frequency $: f_{0}=5.0 \mathrm{GHz}$ \\
Normalized Bandwidth $: w=7.5 \%(@ R L=-10 \mathrm{~dB})$ \\
\hline \hline Transmission line $: Z_{01}=45.5 \Omega, Z_{02}=50.5 \Omega$ \\
$J$-parameter $: J_{01}{ }^{\prime}=0.001912 \Omega^{-1}, J_{02}=0.0058405 \Omega^{-1}$ \\
\hline
\end{tabular}

Fig. 3. Layouts and design values of the slot dipole antennas with bandpass filters in the case of $n=1$ (a) and $n=2$ (b).

These simulation results are in good agreement with transmission line model results. The desired bandwidths are obtained, and good impedance matches between the antenna impedance $Z_{a}$ and the feeder impedance $Z_{0}$ are attained in the passband.

Figs. 6 and 7 show the normalized radiation pattern and the magnetic current in the case of $n=1$, respectively. The directivity looks like that of a typical slot dipole antenna, since it seems that the radiation from the bending parts of the slot is sufficiently small as shown in Fig. 7. The same result was also obtained in the case of $n=2$.

Increasing the pole number $n$, we can obtain wider bandwidth at the sacrifice of the size. With the port number of $n=3$, the bandwidth could be $8.6 \%$ at $R L=-10 \mathrm{~dB}$. 


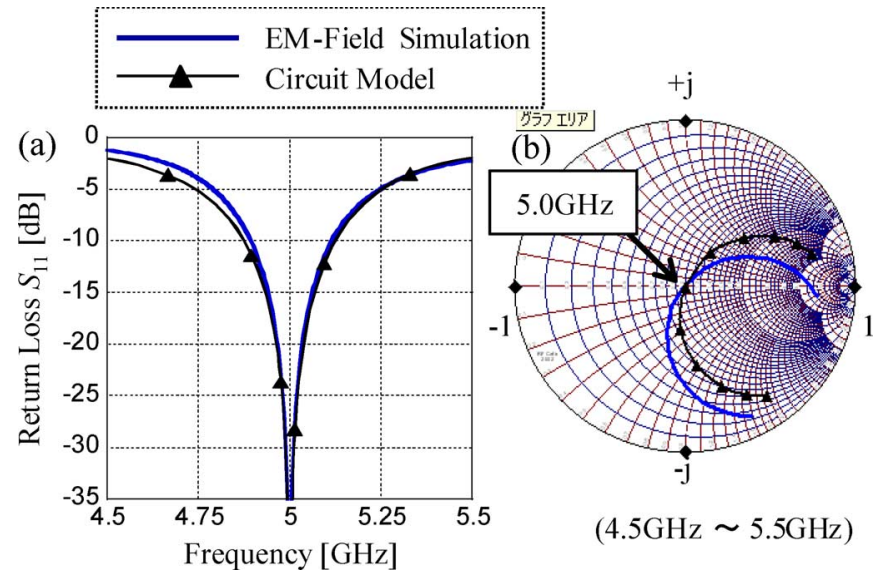

Fig. 4. Frequency dependence of return loss of the slot dipole antenna with a 1-pole bandpass filter [(a) Return Loss, (b) Smith Chart].

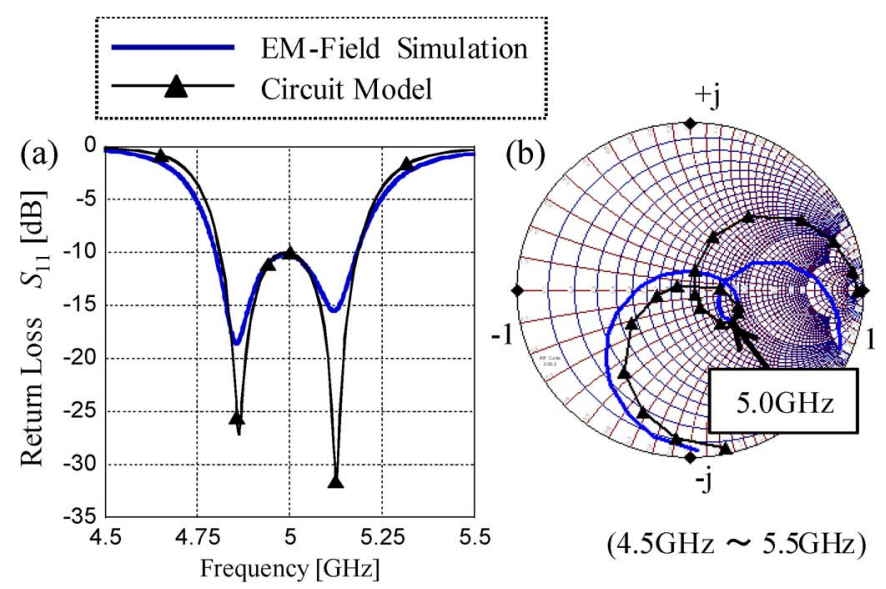

Fig. 5. Frequency dependence of return loss of the slot dipole antenna with a 2-pole bandpass filter [(a) Return Loss, (b) Smith Chart].
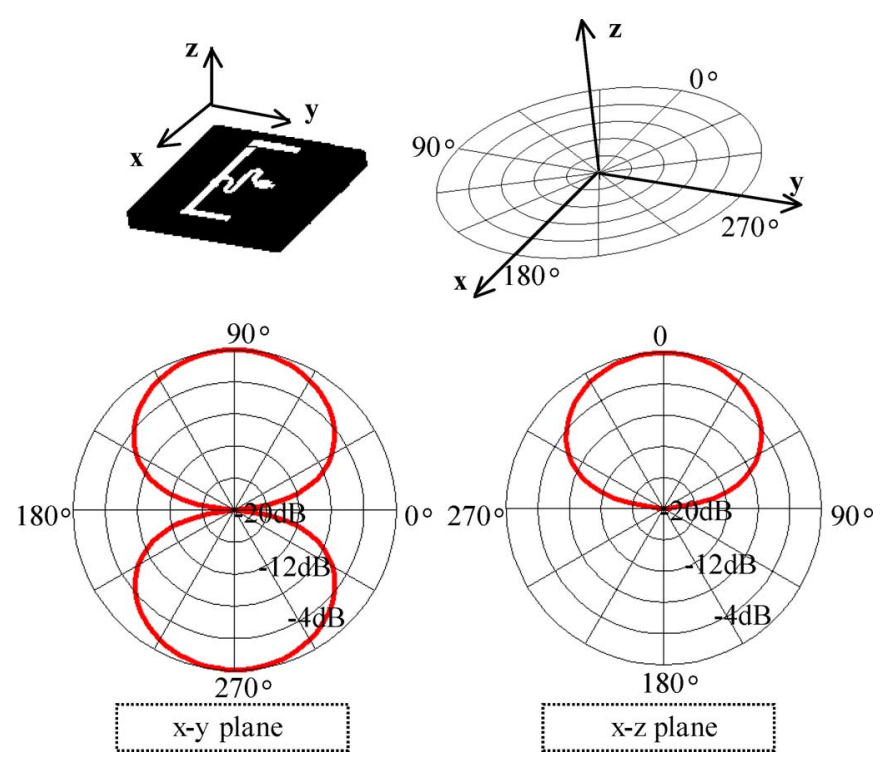

Fig. 6. Directivity pattern of the slot dipole antenna (EM field simulation).

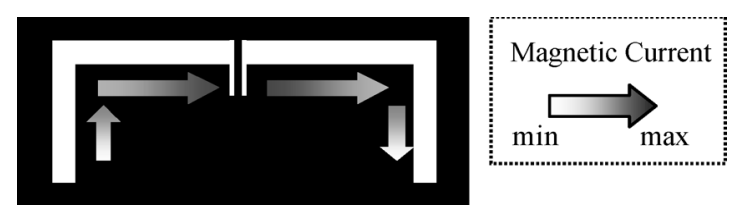

Fig. 7. Magnetic current ofthe slot dipole antenna (EM field simulation).

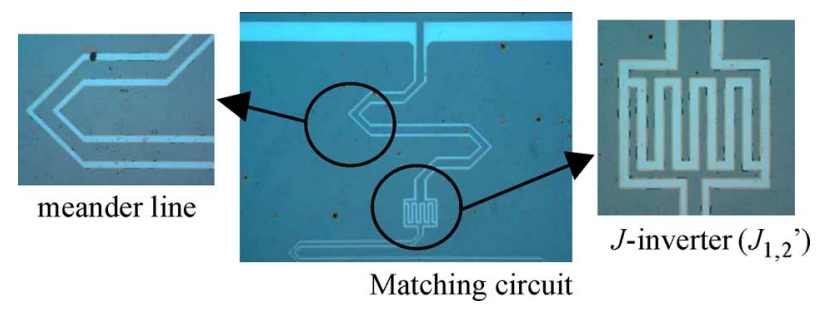

Fig. 8. Photograph of the slot dipole antenna with a 2-pole filter (Substrate: $\mathrm{MgO}$, Film: $\mathrm{YBa}_{2} \mathrm{Cu}_{3} \mathrm{O}_{7-\mathrm{x}}$ ).
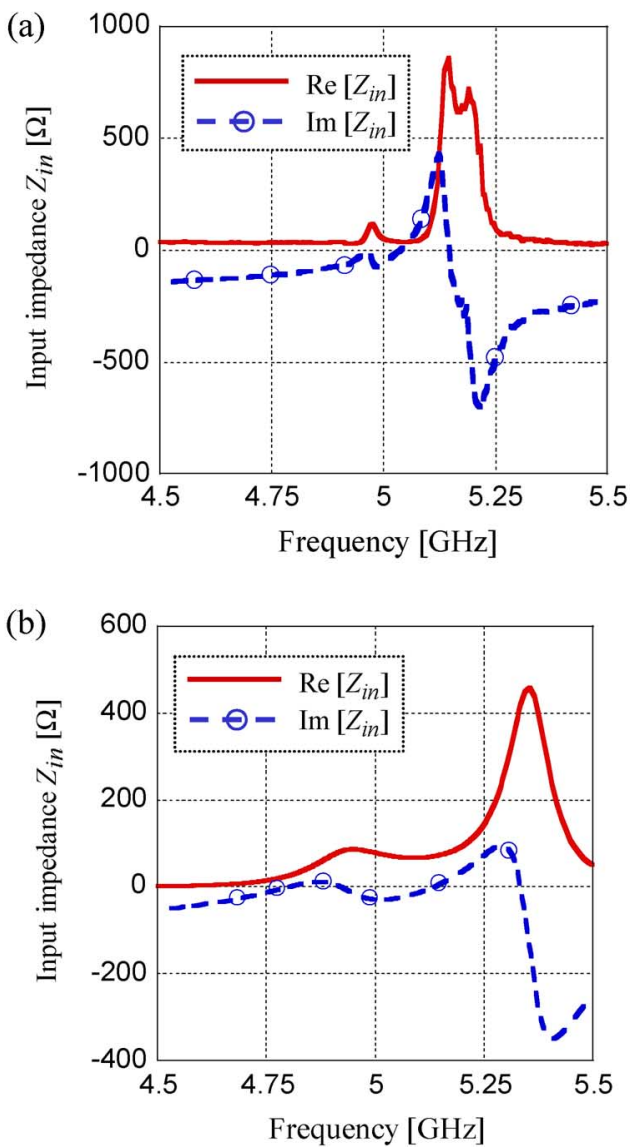

Fig. 9. Obtained frequency characteristic of the input impedance [(a) experimental result, (b) simulation result].

\section{EXPERIMENT}

\section{A. Experimental Procedure}

An Experiment was carried out on the YBCO slot dipole antenna with a 2-pole bandpass filter fabricated by wet etching process, whose layout and photograph are shown in Fig. 3(b) and Fig. 8, respectively. The antenna was placed in a vacuum cryostat, and was measured by using a coplanar waveguide 


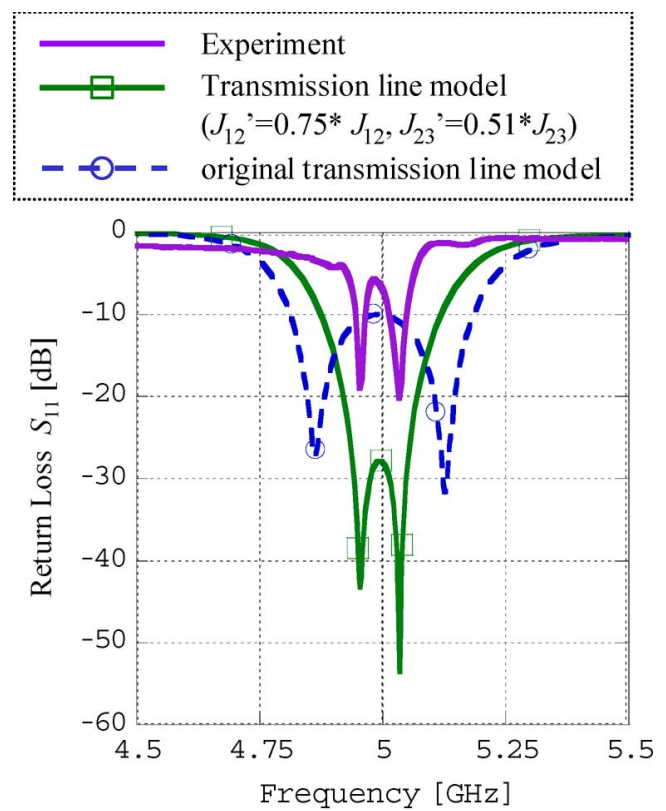

Fig. 10. Comparison of frequency characteristics of the return loss and the calculations taking account of theover etching of $J$-inverters.

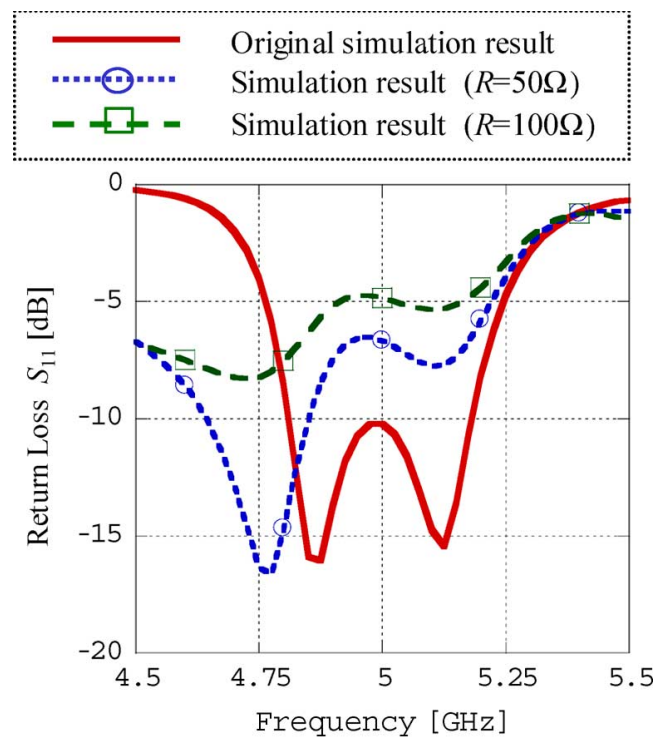

Fig. 11. Comparison of simulated return loss taking account of theresidual loss of a probe.

probes. The frequency response of the $S$-parameter was measured with a vector network analyzer (HP-8722C) at $20 \mathrm{~K}$.

\section{B. Experimental Result}

Fig. 9(a) shows the experimental result for the input impedance of the present miniaturized antenna, which is expressed as $Z_{\text {in }}$ in Fig. 1, and Fig. 9(b) shows the EM-field simulation result.

Fig. 10 shows the experimental result for the return loss and the result of transmission line. It seems that the decrease of the bandwidth observed in the experimental result caused by overetching of $J$-inverters. In Fig. 10, we also plotted the theoretical curve taking account the reduction of $J$-parameters due to overetching. Furthermore, the deterioration of the return loss seems to be caused by a residual loss from the connection of the probe, and this is simulated in Fig. 11, where the simulated return loss taking account of the residual loss of a probe are comparatively illustrated.

However, the observed two peaks which are characteristic of $n=2$ filters are in reasonable agreement to the theoretical performance. Considering experimental errors, it is demonstrated that the designed matching circuit operated as expected.

\section{CONCLUSION}

In this paper, slot dipole antennas with a bandpass filter have been designed and tested. We designed a circuit which matches the small radiation resistance $R_{a}(=3.78 \Omega$ of ESA to the feeder impedance $Z_{0}(=50 \Omega)$. As a result, we designed a small $(4.1 \mathrm{~mm} \times 1.9 \mathrm{~mm})$ and broadband $(R L=-3 \mathrm{~dB}$, $w=13 \%$ ) planar antenna. Moreover, an YBCO slot dipole antenna with a 2-pole bandpass filter was fabricated and tested at a cryogenic temperature, thereby we demonstrated the frequency characteristic as expected.

\section{REFERENCES}

[1] R. Azadegan and K. Sarabandi, "A novel approach for miniaturization of slot antennas," IEEE Trans. Antennas Propagat., vol. 51, no. 3, pp. 421-429, March 2003.

[2] S. C. Gao, L. W. Li, M. S. Leong, and T. S. Yeo, "Dual-polarized slotcoupled planar antenna with wide bandwidth," IEEE Trans. Antennas Propagat., vol. 51, no. 3, pp. 421-429, March 2003.

[3] K. F. Tong, K. Li, T. Matsui, and M. Izutsu, "Broad-band double-layered coplanar patch antennas with adjustable CPW feeding structure," IEEE Trans. Antennas Propagat., pp. 3153-3156, November 2004.

[4] A. Grbic and G. V. Eleftheriades, "Leakly CPW-based slot antenna arrays for millimeter-wave applications," IEEE Trans. Antennas Propagat., vol. 52, no. 11, pp. 3153-3156, November 2004.

[5] M. S. A. Salameh, Y. M. M. Antar, and G. Seguin, "Coplanar-waveguide-fed slot-coupled rectangular dielectric resonator antenna," IEEE Trans. Antennas Propagat., vol. 50, no. 10, pp. 1415-1419, October 2002.

[6] K. Satoh, T. Mimura, S. Narahashi, and T. Nojima, "Today and tomorrow of HTS technology applications," in MWE Microwave Workshop Digest, 2000, pp. 102-117.

[7] S. Ohshima, "High-temperature superconducting passive microwave devices, filters and antennas," Supercond. Sci. Technol., vol. 13, pp. 103-108, January 2000.

[8] K. Yoshida, T. Takahashi, H. Kanaya, T. Uchiyama, and Z. Wang, "Superconducting slot antenna with broadband impedance matching circuit," IEEE Trans. Appl. Supercond., vol. 11, pp. 103-106, March 2001.

[9] H. Kanaya, Y. Koga, J. Fujiyama, G. Urakawa, and K. Yoshida, "Design and performance of high $T_{c}$ superconducting Coplanar waveguide matching circuit for RF-CMOS LNA," IEICE Trans. Electron., vol. E86-C, no. 1, pp. 37-41, January 2003.

[10] Y. Tsutsumi, K. Kanaya, and K. Yoshida, "Design and performance of an electrically small slot loop antenna with a miniaturized superconducting matching circuit," IEEE Trans. Applied Superconductivity, vol. 15, no. 2, pp. 1020-1023, 2005.

[11] K. Yoshida, Y. Tsutsumi, and H. Kanaya, "Electrically small antennas with miniaturized impedance matching circuits for semiconductor amplifiers," IEICE Trans. Electron., vol. E88-C, no. 7, pp. 1368-1374, July 2002.

[12] K. Yoshida, S, Sakaguchi, and H. Kanaya, "Development of a novel impedance matching circuit for electrically small antennas," in Institute of Physics, Conference Series 43, Proc. of 7th EUCAS, 2006, pp. 1362-1365.

[13] G. L. Matthaei, L. Young, and E. M. T. Jones, Microwave Filters, Impedance-Matching Networks, and Coupling Structures. Norwood, MA: Artech House, 1980. 